\title{
Micobiota e micotoxinas em amostras de arroz coletadas durante o sistema estacionário de secagem e armazenamento
}

\author{
Mycobiota and mycotoxins in rice samples collected during the stationary drying and storage system
}

\author{
Michele Hoeltz $^{\mathrm{I}^{*}}$ Carlos Alberto Fagundes $^{\mathrm{II}}$ Eduardo Aléxis Lobo Alcayaga $^{\mathrm{III}}$ Isa Beatriz Noll ${ }^{\mathrm{IV}}$
}

\section{RESUMO}

Considerando as perdas qualitativas e quantitativas no período pós-colheita de grãos, neste trabalho foram avaliadas a contaminação fúngica e as micotoxinas em arroz com casca, durante o sistema estacionário de secagem e armazenamento. As amostras foram coletadas em intervalos de 60 dias durante o armazenamento, em duas alturas do silo-

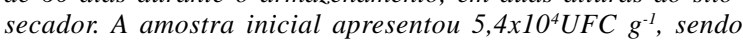
que a contagem aumentou significativamente durante a secagem e o armazenamento, chegando a $10^{5} \mathrm{UFC}^{-1}$. A contaminação fúngica diferiu no interior do silo-secador, com maior contaminação na porção superior do silo. Os gêneros predominantes foram Aspergillus e Penicillium, com maior ocorrência de A. flavus (26,3\%) e P. commune (19,1\%). Quatro isolados de A. flavus produziram aflatoxina $B_{1}$, mas não foram detectadas micotoxinas nas amostras.

Palavras-chave: arroz, secagem, armazenamento, fungos, micotoxinas.

\section{ABSTRACT}

Considering the qualitative and quantitative losses in post-harvest of grain, in this research was evaluated fung and mycotoxins contamination in whole rice (Oryza sativa L.) during the stationary drying and storage system. Samples were collected in a period of sixty days in two heights of the warehouse

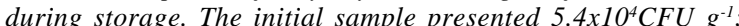
the contamination increased significantly during drying and storage, up to $10^{5} \mathrm{CFU} \mathrm{g}^{-1}$. Fungi contamination was different inside the warehouse with higher contamination in the upper portion. The more abundant fungi genera were Aspergillus and Penicillium, A. flavus (26.3\%) and P. commune $(19.1 \%)$ which presented higher incidence. Aflatoxin $B_{1}$ was produced by four A. flavus isolates, but mycotoxins were not detected in the samples.

Key words: rice, drying, storage, fungi, mycotoxins.

\section{INTRODUÇÃO}

Na América Latina, em países como o Brasil, a Colômbia e o Peru, o arroz (Oryza sativa L.) é um item básico na dieta da população e igualmente importante como produto no comércio internacional (EMBRAPA, 2005). O Brasil ocupa o décimo lugar na produção mundial desse cereal, sendo que o Rio Grande do Sul, maior produtor nacional, contribui com cerca de $60 \%$ da produção estimada para a safra 2007/2008 (CONAB, 2008).

De acordo com dados das Organizações das Nações Unidas para Agricultura e Alimentação (FAO, 2004), $16 \%$ da safra anual de arroz é perdida em decorrência de práticas de pós-colheita ineficientes. A falta de Boas Práticas Agrícolas, bem como as condições tropicais de temperatura e umidade elevadas, propiciam o desenvolvimento fúngico, já que o arroz constitui um ótimo substrato, rico em carboidratos (COELHO et al., 1999).

A atividade fúngica, principalmente durante o armazenamento, pode levar à rápida deterioração na qualidade nutricional dos grãos e à contaminação com micotoxinas (ANDRADE et al., 2003; MAGAN \& ALDRED, 2007).

Micotoxinas são metabólitos secundários tóxicos produzidos principalmente por gêneros Aspergillus, Penicillium e Fusarium, sendo alguns desses compostos potenciais agentes carcinogênicos a humanos e animais (HUSSEIN \& BRASEL, 2001; CALDAS et al., 2002).

\footnotetext{
IPrograma de Pós-graduação em Microbiologia Agrícola e do Ambiente, Universidade Federal do Rio Grande do Sul (UFRGS). Av. Bento Gonçalves 9500, 91570-901, Porto Alegre, RS, Brasil. E-mail: michelehoeltz@yahoo.com.br. *Autor para correspondência. IIInstituto Rio-grandense de Arroz (IRGA), Cachoeirinha, RS, Brasil.

IIIUniversidade de Santa Cruz do Sul (UNISC), Santa Cruz do Sul, RS, Brasil.

${ }^{\mathrm{IV}}$ Instituto de Ciência e Tecnologia de Alimentos, Universidade Federal do Rio Grande do Sul (UFRGS), Porto Alegre, RS, Brasil.
} 
Embora seja menos comum em arroz do que em outros cereais, a contaminação por micotoxinas (aflatoxinas, ocratoxina A, citrinina, sterigmatocistina, fumonisinas e zearalenona) foram encontradas nesse cereal em diversas partes do mundo (FURLONG et al., 1999; LIMA et al., 2000; BIANCHINI, 2003; SIMIONATO et al., 2003; PARK et al., 2005; TANAKA et al., 2007). Recentemente, casos de intoxicação por citreoviridina foram diagnosticados no Estado brasileiro do Maranhão a partir do consumo de arroz contaminado, provocando quadros clínicos de beribéri e levando dezenas de pessoas à morte pela deficiência de vitamina $\mathrm{B}_{1}$ (LIRA \& ANDRADE, 2008).

O número de trabalhos que avalia a contaminação fúngica e por micotoxinas em grãos vem crescendo no Brasil (FURLONG et al., 1999; RODRIGUEZ-AMAYA \& SABINO, 2002; NUNES et al., 2003). Entretanto, pesquisas sobre a qualidade do arroz relacionada a processos pós-colheita ainda são escassas, mesmo tendo-se conhecimento que são, principalmente, durante esses processos que a deterioração e produção de micotoxinas podem ocorrer (BIANCHINI, 2003; JAYAS \& WHITE, 2003; MAGAN \&ALDRED, 2007).

O objetivo deste trabalho foi avaliar a contaminação por fungos e micotoxinas em arroz com casca, durante o processo de secagem estacionária, empregando gás liquefeito de petróleo (GLP) e armazenamento no silo-secador.

\section{MATERIAL E MÉTODOS}

O trabalho foi realizado em parceria com o Instituto Rio-grandense do Arroz (IRGA), situado na cidade de Cachoeirinha, Rio Grande do Sul, (RS), Brasil. Os grãos foram depositados no interior de um silo metálico cilíndrico com capacidade para sete toneladas de arroz com casca (3,0m de altura e 2,8m de diâmetro). Durante a secagem, o ar aquecido pela queima de gás liquefeito de petróleo (GLP), com temperatura de aproximadamente $40^{\circ} \mathrm{C}$, foi insuflado pela parte inferior do silo (fundo falso). Após os grãos atingirem umidade de $13 \%$, o sistema foi desligado, totalizando um período de secagem de 30 dias. O produto foi então armazenado dentro do próprio silo por 12 meses. Quando umidade ou temperatura no interior do silo-secador ultrapassava $70 \%$ e $19^{\circ} \mathrm{C}$, respectivamente, o ar ambiente era automaticamente insuflado. A fim de controlar fatores como temperatura e umidade interna do silo e da massa de grãos, foi utilizado um sistema computadorizado de controle, cedido pela empresa Dryeration Indústria, Comércio, Projetos e Representações LTDA. Com esse sistema, as condições foram monitoradas diariamente durante o período da pesquisa, sendo os dados coletados em intervalos de 15 minutos. Para análise estatística, foram consideradas as médias desses dados. A análise da umidade do arroz foi realizada segundo método sugerido pelas Regras de Análises de Sementes (MAARA, 1992) em estufa a $105^{\circ} \mathrm{C}$.

Uma amostra inicial $\left(\mathrm{T}_{0}\right)$ foi coletada, representando os grãos recém-colhidos. Durante a secagem, foram coletadas duas amostras, aos $15\left(\mathrm{~T}_{15}\right)$ e 30 dias $\left(\mathrm{T}_{30}\right)$. Durante o armazenamento, as amostragens foram realizadas a cada dois meses. As 36 amostras de $1 \mathrm{~kg}$ foram coletadas com um amostrador pneumático manual em duas alturas do silo-secador: terço inferior $(0,40 \mathrm{~m}$ a partir do fundo falso) e terço superior (1,8m a partir do fundo falso) em duplicata, acondicionadas em saco plástico esterilizado e imediatamente levadas ao Laboratório de Toxicologia do Instituto de Ciência e Tecnologia de Alimentos UFRGS para análise.

A contagem total de bolores e leveduras foi realizada pela técnica de plaqueamento em superfície, em Agar Batata Dextrose Acidificado, sendo que as amostras foram incubadas a $25^{\circ} \mathrm{C}$ por cinco dias (ASSOCIAÇÃO BRASILEIRA DE NORMAS TÉCNICAS - MB - 2750). Colônias morfologicamente distintas foram isoladas em Ágar Sabouraud (Biobrás) e reincubadas a $25^{\circ} \mathrm{C}$ (LACAZ, 1991). Espécies de Aspergillus e Penicillium foram identificadas conforme PITT \& HOCKING (1997).

Para a avaliação do potencial aflatoxigênico, as colônias de Aspergillus isoladas foram cultivadas em Ágar Coco e incubadas a $25^{\circ} \mathrm{C}$ por sete dias. Após raspagem do micélio, o meio foi cortado em tiras, macerado em $1 \mathrm{~mL}$ de clorofórmio, e o extrato foi analisado por Cromatografia em Camada Delgada (CCD) (LIN \& DIANESE, 1976). As aflatoxinas foram confirmadas ao borrifar $\mathrm{H}_{2} \mathrm{SO}_{4}$ a $25 \%$ e derivatizar com ácido trifluoracético (PRZYBYLSKI, 1975).

A determinação de aflatoxinas $B_{1}, B_{2}, G_{1}$ e $\mathrm{G}_{2}$, ocratoxina A e zearalenona foi feita por CCD, de acordo com o método descrito por SOARES \& RODRIGUEZ-AMAYA (1989). Após extração com metanol/KCl 4\% (9:1), clarificação e partição líquidolíquido com clorofórmio, o extrato seco foi redissolvido em $100 \mu \mathrm{L}$ de clorofórmio e aplicado juntamente com os padrões de micotoxinas em cromatofolhas de alumínio 60G (Merck, Alemanha). Após a eluição em tolueno: acetato de etila: clorofórmio: ácido fórmico (35:25:25:10), foi realizada a leitura sob luz ultravioleta de $365 \mathrm{~nm}$. Os padrões utilizados nas análises foram da marca Sigma Chemical (EUA), e o preparo desses padrões foi realizado de acordo com as recomendações $970.44 \mathrm{e}$ 971.22 da AOAC (1990), sendo que foram estabelecidas 
as concentrações de trabalho de $8 \mu \mathrm{g} \mathrm{mL} \mathrm{mL}^{-1}$ para as aflatoxinas e a ocratoxina $\mathrm{A}$ e $40 \mu \mathrm{g} \mathrm{mL} \mathrm{m}^{-1}$ para zearalenona. Os limites de detecção encontrados foram de $3 \mu \mathrm{g} \mathrm{kg}^{-1}$ para aflatoxinas $\mathrm{B}_{1}$ e $\mathrm{G}_{1}, 1 \mu \mathrm{g} \mathrm{\textrm {kg } ^ { - 1 }}$ para aflatoxinas $\mathrm{B}_{2} \mathrm{e} \mathrm{G}_{2}, 5 \mu \mathrm{g} \mathrm{kg}{ }^{-1}$ para ocratoxina A e $110 \mu \mathrm{g}$ $\mathrm{kg}^{-1}$ para zearalenona.

Os resultados de umidade e temperatura coletados foram analisados quanto as suas diferenças pela prova estatística não-paramétrica "U" de MannWhitney (SIEGEL, 1975). Para avaliar a influência dos fatores sobre a contaminação fúngica, foi aplicada uma análise de regressão linear múltipla, utilizando o programa GraphPad InStat ${ }^{\circledR}$ ver. 3.00 para Windows 95/NT.

\section{RESULTADOS E DISCUSSÃO}

A contagem total de bolores na amostra inicial ( $\mathrm{T}$ ) foi de $5,4 \times 10^{4} \mathrm{UFC}^{-1}$. Durante o processo de secagem $\left(T_{15}\right.$ e $\left.T_{30}\right)$, já foi observado um aumento significativo da contagem, atingindo nível de $10^{5} \mathrm{UFC}$ $\mathrm{g}^{-1}$, sendo que tal valor se manteve durante seis meses de armazenamento. Somente nas análises feitas aos oito, 10 e 12 meses foi observada a redução de 1 log, voltando a se comparar com a amostra inicial ( $\mathrm{T}_{0}$ ) (Figura 1).

Esses resultados estão de acordo com ELIAS (2000), o qual cita que o sistema estacionário, da forma em que foi empregado neste trabalho, é extremamente dependente de condições psicométricas de difícil controle e um agente facilitador da deterioração dos grãos de arroz por microrganismos.

Resultados comparativos entre as amostragens feitas das duas alturas do silo-secador podem ter influenciado os dados acima citados. Como pode se observado ainda na figura 1 , a contagem de bolores no terço inferior do silo-secador apresentou uma média de $4,3 \times 10^{4} \mathrm{UFC} \mathrm{g}^{-1}$, não sendo uma diferença significativa quando comparada à amostra inicial, entretanto, o terço superior apresentou um aumento significativo de contaminação durante o período analisado, apresentando uma média de $3,2 \times 10^{5} \mathrm{UFC} \mathrm{g}^{-1}$ de amostra.

Ao comparar diferentes condições de secagem e armazenamento do arroz em silo metálico, BIANCHINI (2003) também observou uma diferença significativa em relação à enumeração fúngica entre duas alturas do silo-secador. Nesse experimento, o terço superior apresentou maior contaminação em determinados períodos, quando comparado ao terço inferior.

Os gêneros fúngicos isolados das amostras foram Aspergillus, Penicillium, Fusarium, Cladosporium, Phoma, Trichoderma e Rhizopus. Os resultados do presente estudo estão de acordo com

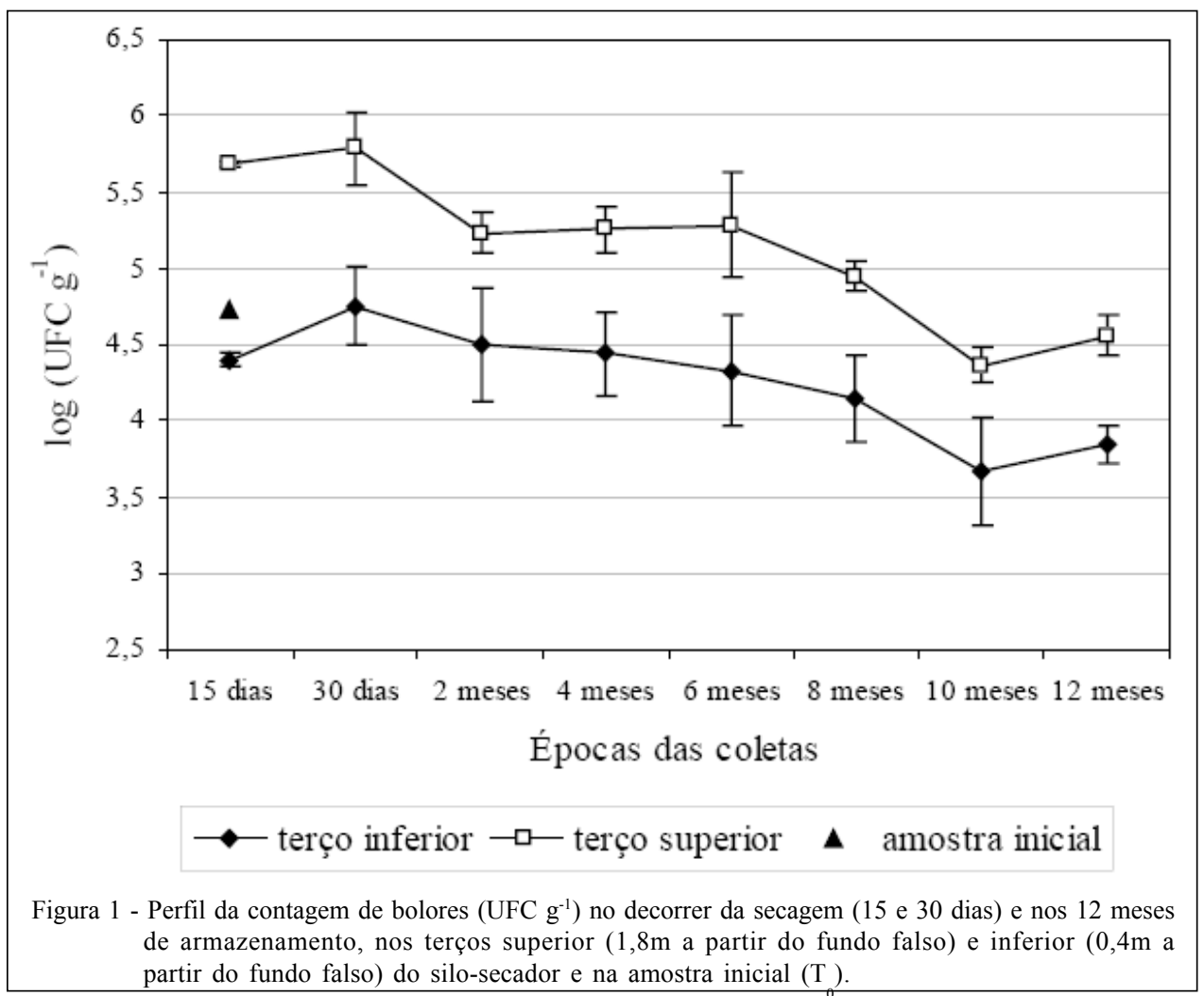

Ciência Rural, v.39, n.3, mai-jun, 2009. 
várias pesquisas realizadas com diversos cereais além do arroz, sendo os três primeiros gêneros os mais citados (FILTENBORG et al., 1996; LIMA et al., 2000; GARCIA et al., 2002; BIANCHINI, 2003; NUNES et al., 2003; PARK et al., 2005).

A amostra inicial $\left(\mathrm{T}_{0}\right)$, com 47 isolados, apresentou maior ocorrência de Aspergillus spp. (65\%), destacando-se A. flavus com 51,3\%, seguido de Penicillium spp.(35\%). A partir da secagem e ao longo do período de armazenamento, com 162 isolados, ocorreu um decréscimo de Aspergillus spp. (34\%) e um aumento de Penicillium spp.(66\%). Quanto às espécies identificadas de todas as amostras, A. flavus (26,3\%) e P. commune (19,1\%) predominaram, seguidas de A. parasiticus (11,9\%), P. corylophilum (9,6\%), $\boldsymbol{P}$. islandicum (7,6\%), P. griseofulvum (7,6\%), A. oryzae (5,4\%), A. japonicus (3,8\%), P. solitum (2,4\%), A. niger $(1,9 \%), \boldsymbol{P}$ variable $(1,4 \%)$, A. fumigatus $(1,4 \%), \boldsymbol{P}$. citrinum $(0,5 \%), \boldsymbol{P}$. canescens $(0,5 \%)$ e $\boldsymbol{P}$. waksmanii $(0,5 \%)$, comumente encontradas em alimentos, sementes e grãos de cereais (PITT \& HOCKING, 1997).

De um total de 55 isolados de A. flavus, apenas quatro cepas $(7,3 \%)$ mostraram-se produtoras de aflatoxina $\mathrm{B}_{1}$ Tal percentual é baixo, considerandose o potencial produtor dessa espécie (ORDAZ et al., 2003). PARK et al. (2005), analisando arroz polido, encontraram $13,3 \%$ dos 15 isolados produtores de aflatoxina $\mathrm{B}_{1}$. LIMA et al. (2000), em suas pesquisas, encontraram 52,6\% de A. flavus aflatoxigênicos, trabalhando com apenas 19 isolados.

Os resultados sugerem que o sistema estacionário de secagem e armazenamento pesquisado neste estudo apresenta problemas no controle de fatores psicométricos. $\mathrm{O}$ aumento nas contagens de bolores se deve, em grande parte, à ineficiência da secagem empregada, que deixou os grãos no terço superior (1,8m a partir do fundo falso) do silo-secador com umidade mais elevada (Figura 2), fator que, ao ser analisado em conjunto com a temperatura, influenciou positivamente a contaminação fúngica (Tabela 1). A retirada de umidade dos grãos e das sementes, sem afetar suas qualidades química e física, é um dos grandes problemas citado por ANDRADE et al. (2003), que aponta a secagem como o principal fator para a manutenção da qualidade dos grãos armazenados.

Ao verificar a influência da temperatura interna do silo-secador, pode-se observar que, no terço inferior $(0,4 \mathrm{~m}$ a partir do fundo falso), esse fator apresentou uma correlação negativa em relação à contaminação fúngica, significando que, na medida em que a temperatura aumentava, a contaminação fúngica decrescia (Figura 3). A mesma correlação negativa entre temperatura e contaminação fúngica foi encontrada por BIANCHINI (2003), pesquisando a influência de sistemas de armazenamento de arroz em silo-secador.

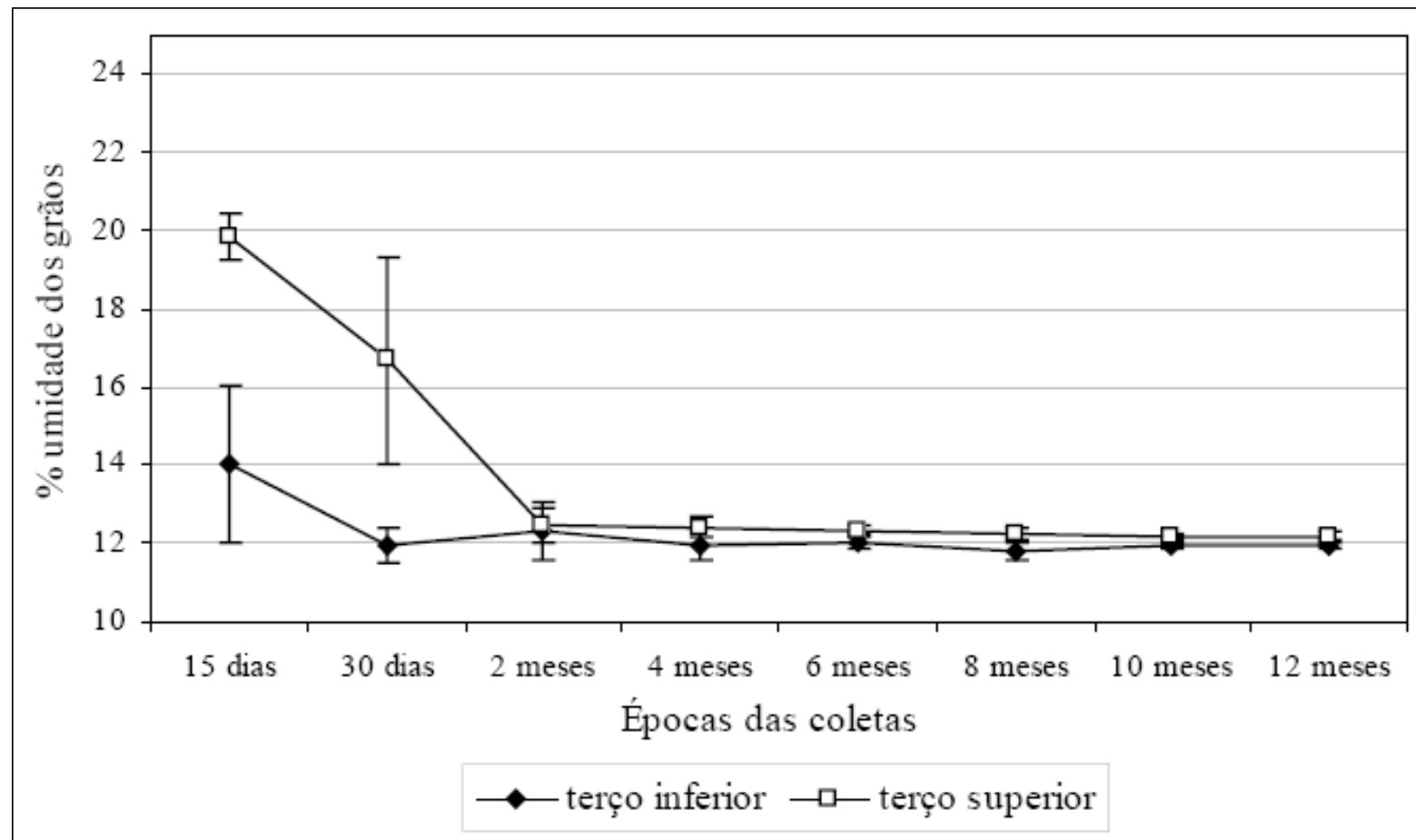

Figura 2 - Perfil da umidade dos grãos (\%) no decorrer da secagem (15 e 30 dias) e nos 12 meses de armazenamento, nos terços superior ( $1,8 \mathrm{~m}$ a partir do fundo falso) e inferior $(0,4 \mathrm{~m}$ a partir do fundo falso). 
Tabela 1 - Análise de regressão linear múltipla para a contagem de bolores (UFC $\mathrm{g}^{-1}$ ) no terço superior (1,8m a partir do fundo falso) do silosecador, tendo como variáveis temperatura $\left({ }^{\circ} \mathrm{C}\right)$ e umidade da massa de grãos $(\%)$.

\begin{tabular}{lcccc}
\hline & Coeficiente & Desvio padrão & Razão T (*) & Valor P \\
\hline Contaminação Fúngica & 5,255 & 1,550 & 3,389 & 0,0195 \\
Temperatura & $-0,09997$ & 0,07438 & 1,344 & 0,2367 \\
Umidade & 0,1453 & 0,06858 & 2,118 & 0,0877 \\
\hline
\end{tabular}

$(*)=$ Coeficiente/Desvio padrão.

Assim como poucos trabalhos têm encontrado contaminação por micotoxinas no arroz, em diferentes níveis e tipos de produtos (FURLONG et al., 1999; LIMA et al., 2000; BIANCHINI, 2003; SIMIONATO et al., 2003; TANAKA, et al., 2007), neste trabalho não foram detectadas aflatoxinas, ocratoxina A e zearalenona em nenhuma das amostras analisadas de arroz com casca. Segundo FILTENBORG et al. (1996), o conjunto das condições encontradas em sistemas de armazenamento nem sempre favorecem a produção de metabólitos tóxicos pelos fungos contaminantes.

\section{CONCLUSÕES}

O sistema estacionário de secagem e armazenamento pesquisado neste trabalho deve ser reavaliado quanto a sua eficiência, uma vez que houve um aumento significativo da contagem de bolores durante o período de análise. Nesse sentido, a umidade dos grãos representou influência positiva na contaminação fúngica do arroz armazenado no terço superior do silo-secador, enquanto que no terço inferior a temperatura interna do silo-secador influenciou negativamente a contaminação. Durante a secagem e o armazenamento, espécies de gênero Penicillium (66\%) foram mais abundantes, seguidas por espécies de Aspergillus (34\%). Embora tenha sido verificada a presença de linhagens de A. flavus aflatoxigênicas $(7,3 \%)$, não foram detectadas aflatoxinas ou outras micotoxinas nas amostras analisadas. Com os resultados deste trabalho, evidencia-se a importância e necessidade do emprego das Boas Práticas Agrícolas, principalmente em manejos pós-colheita, evitando o desenvolvimento de fungos potencialmente toxigênicos e mantendo a integridade dos grãos.

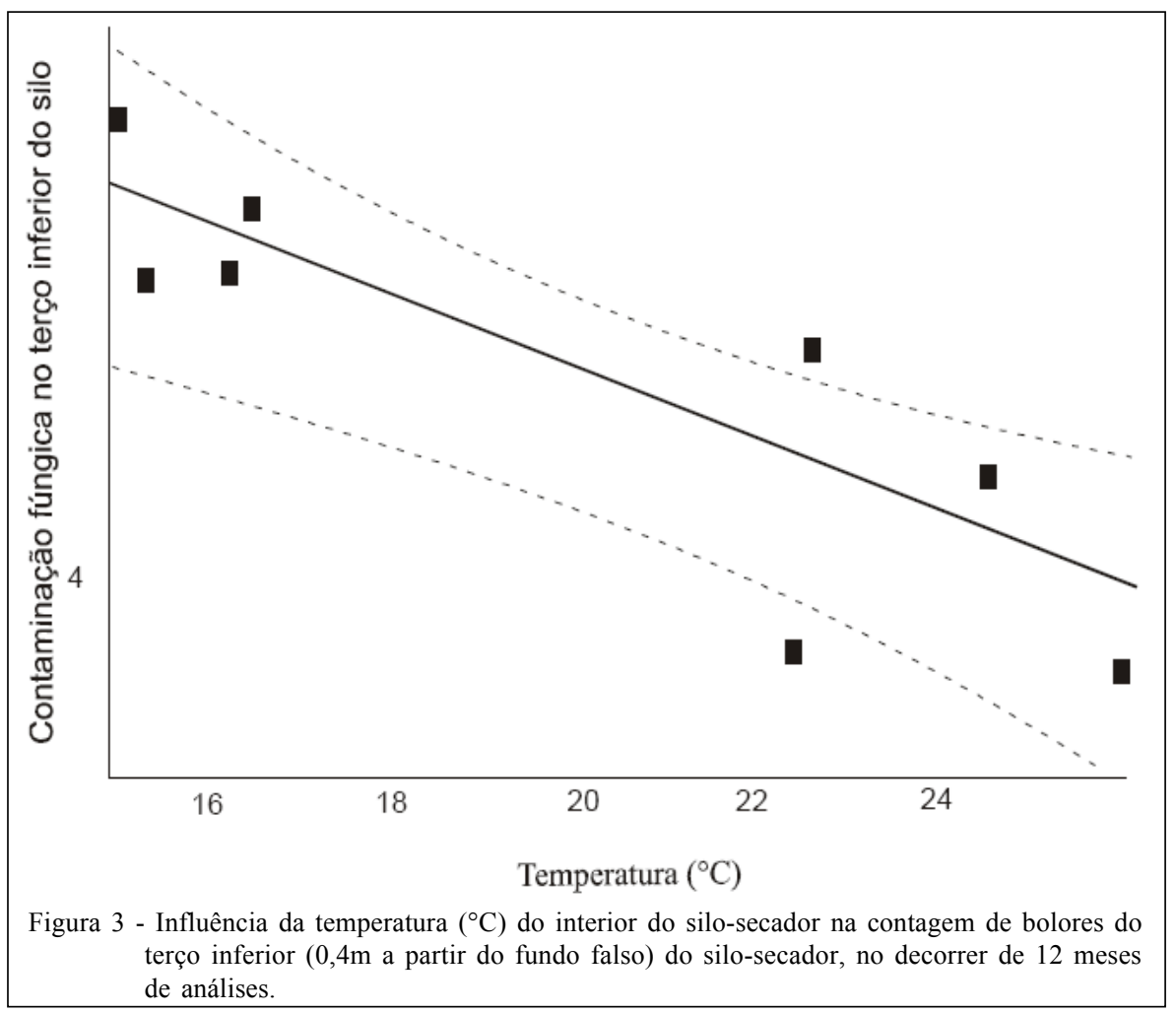

Ciência Rural, v.39, n.3, mai-jun, 2009. 


\section{AGRADECIMENTOS}

Os autores agradecem à Coordenação de Aperfeiçoamento de Pessoal de Nível Superior (CAPES), pela bolsa de pós-graduação concedida, ao Programa de Pósgraduação em Microbiologia Agrícola e do Ambiente-UFRGS e ao Instituto de Ciência e Tecnologia de Alimentos-UFRGS, pela estrutura disponibilizada, ao Instituto Rio Grandense do arroz (IRGA), pela parceria realizada e por todas as análises prestadas, e à Dryeration, pela implantação de um sistema automatizado de leituras no silo testado.

\section{REFERÊNCIAS}

ASSOCIAÇÃO BRASILEIRA DE NORMAS TÉCNICAS ABNT. Manual de métodos de análise microbiológica de alimentos. São Paulo: Varela, 1997. 295p.

ANDRADE, E.T.et al. Qualidade de sementes de milho armazenadas em silo metálico cilíndrico. Revista Brasileira de Armazenamento, v.28, p.23-30, 2003.

BIANCHINI, A. Estudo comparativo entre manejos de secagem e armazenamento de arroz na incidência de fungos toxigênicos e micotoxinas. 2003. 103f. Dissertação (Mestrado em Microbiologia Agrícola e do Ambiente) - Curso de Pós-graduação em Microbiologia Agrícola e do Ambiente, Universidade Federal do Rio Grande do Sul.

CALDAS, E.D. et al. Aflatoxinas e ocratoxina A em alimentos e riscos para a saúde humana. Revista de Saúde Pública, São Paulo, v.36, n.3, p.319-323, 2002.

COELHO, C.S.P. et al. Migração de micotoxinas durante a parboilização do arroz. Brazilian Journal of Food Technology, v.1, n.2, p.39-44, 1999.

COMPANHIA NACIONAL DE ABASTECIMENTO - CONAB. Acompanhamento da safra brasileira: grãos. Brasília, 2008. Capturado em 05 mai 2008. On line. Disponível na Internet http://www.conab.gov.br/conabweb/download/safra

ELIAS, M.C. Tecnologia em secagem e armazenamento de grãos: sistemas, processos e métodos. Pelotas: Pólo de Inovação Tecnológica em Alimentos da Região Sul, Conselho Regional de Desenvolvimento da Região Sul (Corede-Sul), 2000. $84 \mathrm{p}$.

EMPRESA BRASILEIRA DE PESQUISA - EMBRAPA. Cultivo do arroz irrigado no Brasil. Pelotas, 2005. Capturado em 05 mai. 2008. On line. Disponível na Internet http://sistemasdeproducao.cnptia.embrapa.br/FontesHTML/ Arroz/ArrozIrrigadoBrasil/index.htm

FILTENBORG, O. et al. Moulds in food spoilage. International Journal of Food Microbiology, v.33, p.85$102,1996$.

FURLONG, E.B. et al. Aflatoxinas, ocratoxina A e zearalenona em alimentos da região sul do Rio Grande do Sul. Revista do Instituto Adolfo Lutz, São Paulo, v.58, n.2, p.105-111, 1999.

GARCIA, M.J.M. et al. Sucessão de espécies de fungos em milho armazenado em sistema aerado. Revista Brasileira de Armazenamento, v.27, n.2, p.14-22, 2002.

HUSSEIN, H.S.; BRASEL, J.M. Toxicity, metabolism, and impact of mycotoxins on humans and animals. Toxicology, v.167, p.101-134, 2001.

JAYAS, D.S.; WHITE, N. D.G. Storage and drying of grain in Canada: low cost approaches. Food Control, v.14, p.255$261,2003$.
LACAZ, C.S. Microbiologia médica. 8 ed. São Paulo: Savier, 1991.

LIMA, C.A.P. et al. Mycoflora and aflatoxigenic species in derivates of milled rice. Ciência e Tecnologia de Alimentos, v.20, n.1, p.37-39, 2000.

LIN, M.T.; DIANESE, J.C. A coconut-agar medium for rapid detection of aflatoxin producing by Aspergillus spp. Phytopathology, v.60, n.12, p.1466-1469, 1976.

LIRA, P.I.C.; ANDRADE, S.L.L.S. Epidemia de beribéri na Maranhão, Brasil. Cadernos de Saúde Publica, v.24, n.6, doi: 10.1590/S0102-311X2008000600001, 2008.

MAGAN, N.; ALDRED, D. Post-harvest control strategies: Minimizing mycotoxins in the food chain. International Journal of Food Microbiology, v.119, p.131-139, 2007.

MINISTÉRIO DA AGRICULTURA, DO ABASTECIMENTO E DA REFORMA AGRÁRIA. Comissão Técnica de Normas e Padrões. Regras para análises de sementes, Brasília, 1992. p.365.

NUNES, I.T. et al. Arroz comercializado na região sul do Brasil: aspectos micotoxicológicos e microscópicos. Ciência e Tecnologia de Alimentos, v.23, n.2, p.190-194, 2003.

ORDAZ, J.J. et al. Development of a method for direct visual, determination of aflatoxin production by colonies of Aspergillus flavus group. International Journal of Food Microbiology, v.83, p.219-225, 2003.

ORGANIZAÇÃO DAS NAÇÕES UNIDAS PARA AGRICULTURA E ALIMENTAÇÃO - FAO. Rice and postharvest production. 2004. Capturado em 02 mai. 2008. On line. Disponível na Internet http://www.fao.org/rice2004/en/ rice6.htm

PARK, J. W. et al. Fungal mycoflora and mycotoxins in Korean polished rice destined for humans. International Journal of Food Microbiology, v.103, p.305-314, 2005.

PITT, J.I.; HOCKING, A. Fungi and food spoilage. London: Chapman \& Hall, 1997. 593p.

PRZYBYLSKI, W. Formation of aflatoxin derivatives on thin layer chromatographic plates. Journal of Association of Official Analytical Chemists, v.58, p.163-164, 1975.

RODRIGUEZ-AMAYA, D.B.; SABINO, M. Mycotoxin research in Brazil: the last decade in review. Brazilian Journal of Microbiology, v.33, p.1-11, 2002.

SIEGEL, S. Estatística não-paramétrica. São Paulo: MacGrwHill do Brasil, 1975. 350p.

SIMIONATO, E.M.R.S. et al. Ocorrência de ocratoxina A e aflatoxinas em arroz. Revista do Instituto Adolfo Lutz, v.62, n.2, p.123-130, 2003.

SOARES, L.M.V; RODRIGUEZ-AMAYA, D.B. Survey of aflatoxins, ochratoxin A, zearalenone and sterigmatocystin in some Brazilian foods by using multi-toxin thin-layer chromatographic method. Journal of Association of Official Analytical Chemists, v.72, n.1, p.22-26, 1989.

TANAKA, K. et al. Mycotoxins in rice. International Journal of Food Microbiology, v.119, p.59-66, 2007. 